\title{
Nanoparticles from Cuttlefish Ink Inhibit Tumor Growth by Synergizing Immunotherapy and Photothermal Therapy
}

Rong-Hui Deng, ${ }^{\ddagger}$ Mei-Zhen Zou, ${ }^{\dagger}$ Diwei Zheng, ${ }^{\dagger}$ Si-Yuan Peng, ${ }^{\dagger}$ Wenlong Liu, ${ }^{\dagger}$ XueFeng Bai,${ }^{\dagger}$ Han-Shi Chen,${ }^{\dagger}$ Y unxia Sun,${ }^{\dagger}$ Pang-Hu Zhou,,$\stackrel{\dagger}{\dagger}$ and Xian-Zheng Zhang*,†

†Key Laboratory of Biomedical Polymers of Ministry of Education, Institute for Advanced Studies (IAS) and Department of Chemistry, Wuhan University, Wuhan 430072, P. R. China

\#Department of Orthopedics, Renmin Hospital of Wuhan University, Wuhan 430060, P. R. China

\footnotetext{
*Corresponding Authors. E-mails: zhoupanghu@126.com (P.-H. Zhou) and xzzhang@whu.edu.cn. (X.-Z.Zhang)

R.-H. Deng and M.-Z. Zou contributed equally to this work.
} 

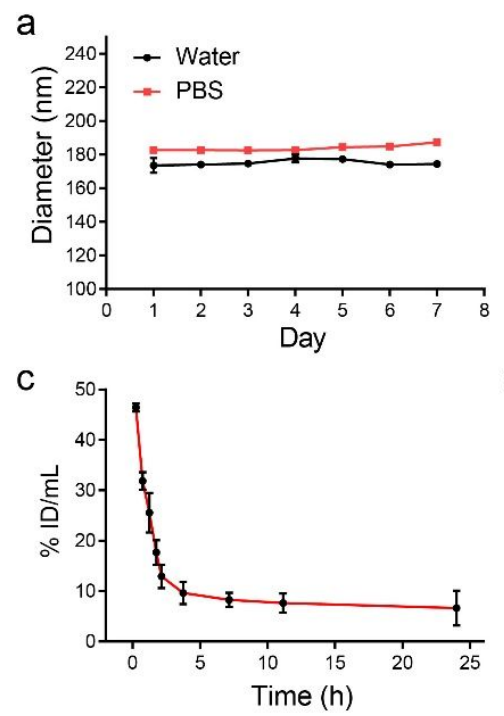
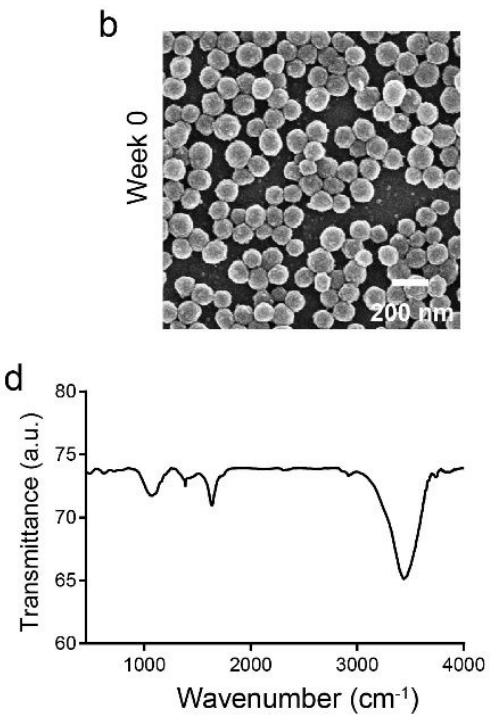

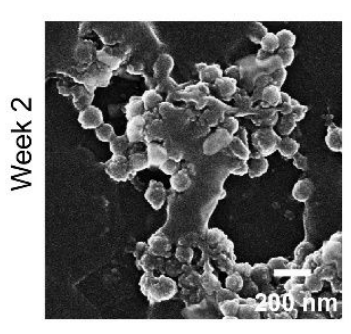

e

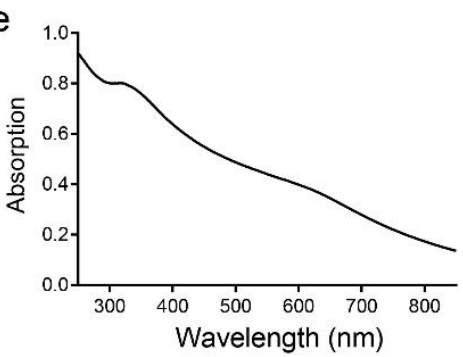

Figure S1. a) The colloidal stability of CINPs in water and PBS. b) SEM images of CINPs after incubation with liver lysates c) Blood circulation curve of CINPs in mice by measuring the fluorescence of Cy5-labeled CINPs in blood at different time points postinjection. d) FT-IR spectrum of CINPs. e) UV-vis spectrum of CINPs in water. 
a

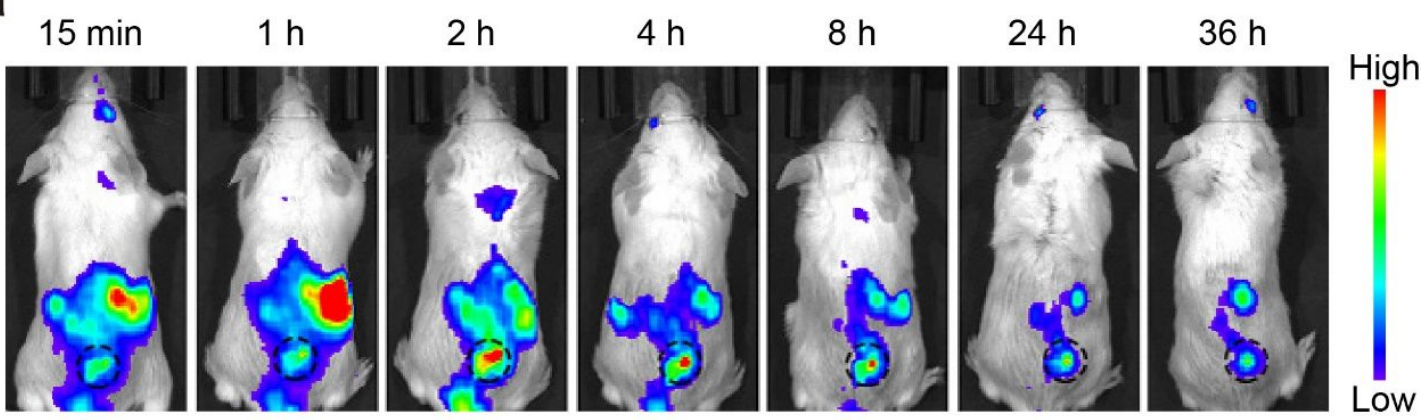

b

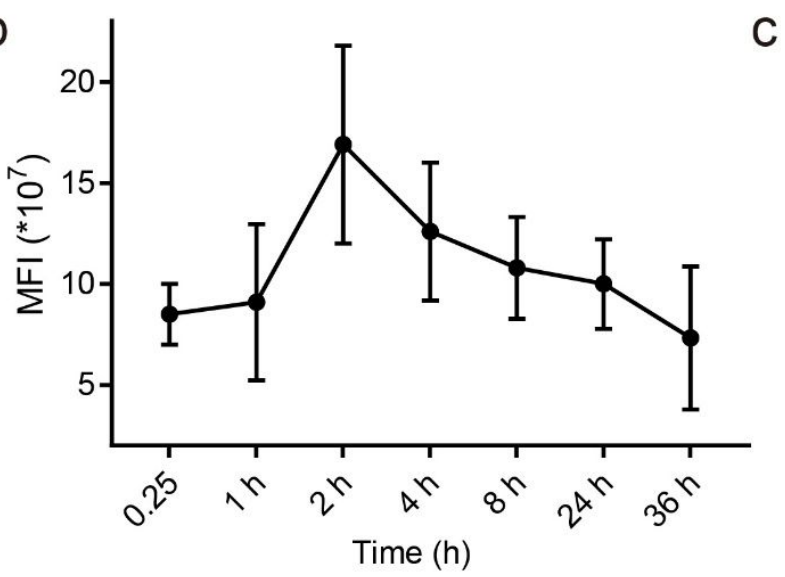

C

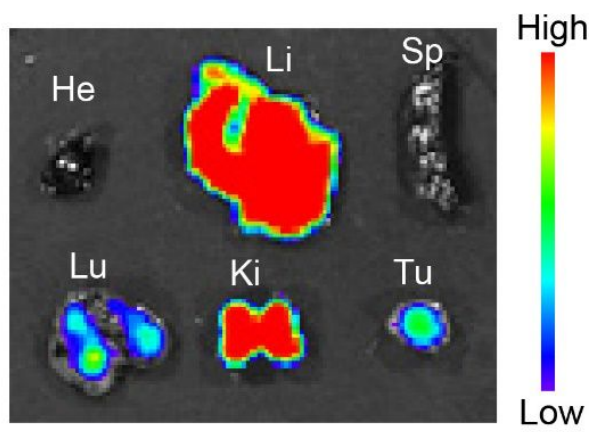

Figure S2. a) In vivo fluorescence images of CT26 tumor bearing mice after intravenously injection of Cy5-labeled CINPs at different times. b) Corresponding quantitative analysis of fluorescence intensity. c) Ex vivo fluorescence images of major organs after $36 \mathrm{~h}$ postinjection (He: heart, Li: liver, Sp: spleen, Lu: lung, Ki: kidney, and Tu: tumor). 
a

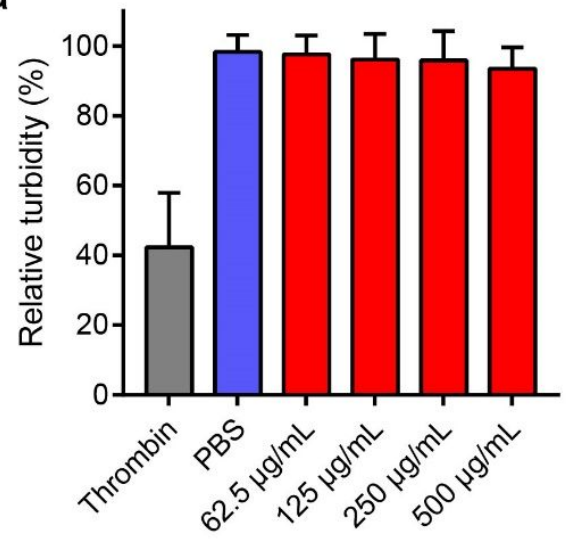

b

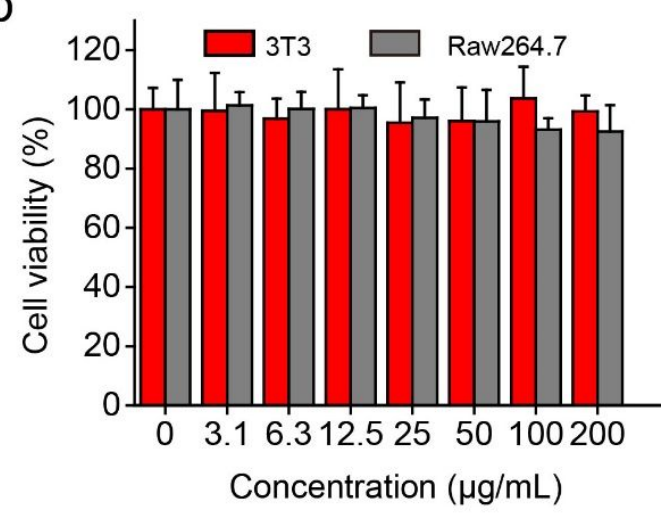

Figure S3. a) Platelet aggregation assay of CINPs. b) Cytotoxicity of CINPs in normal cells 


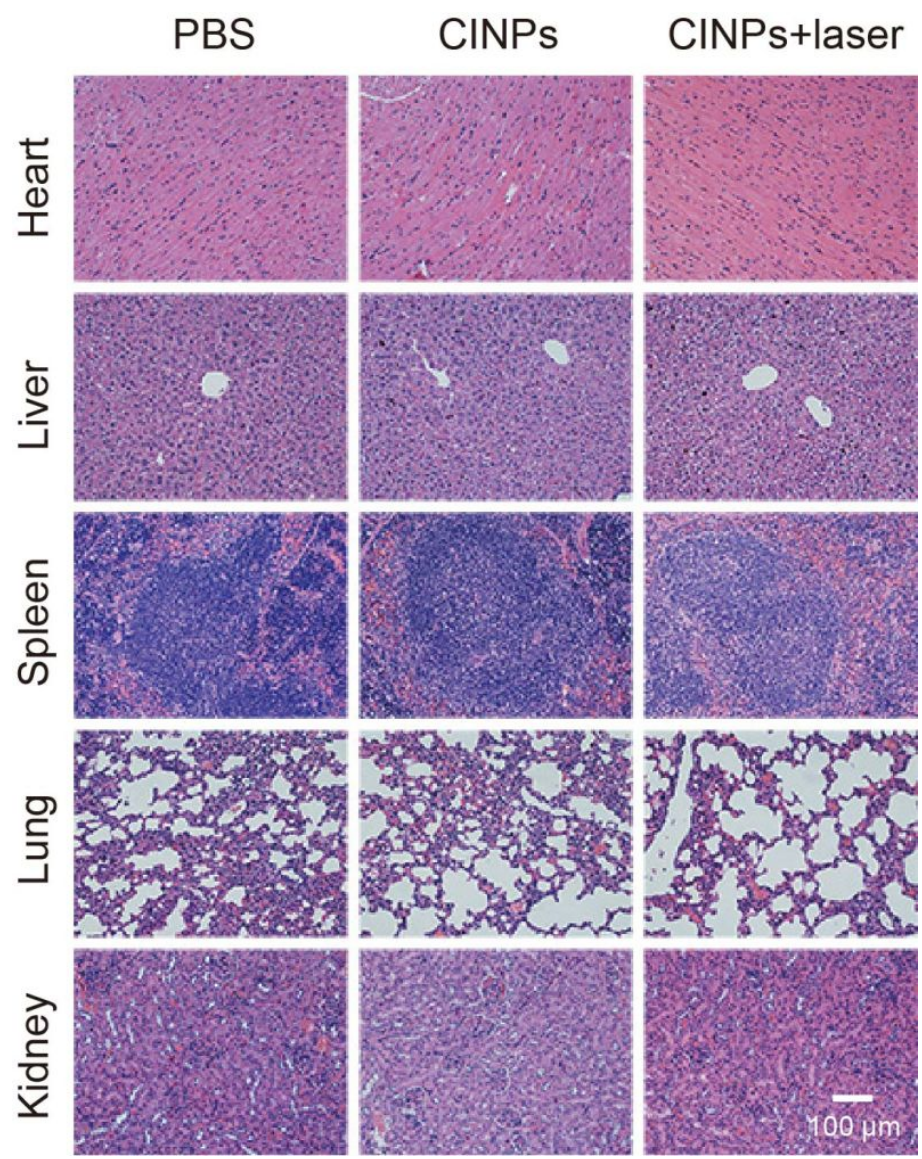

Figure S4. H\&E staining of mice hearts, livers, spleens, lungs and kidneys in different groups. 

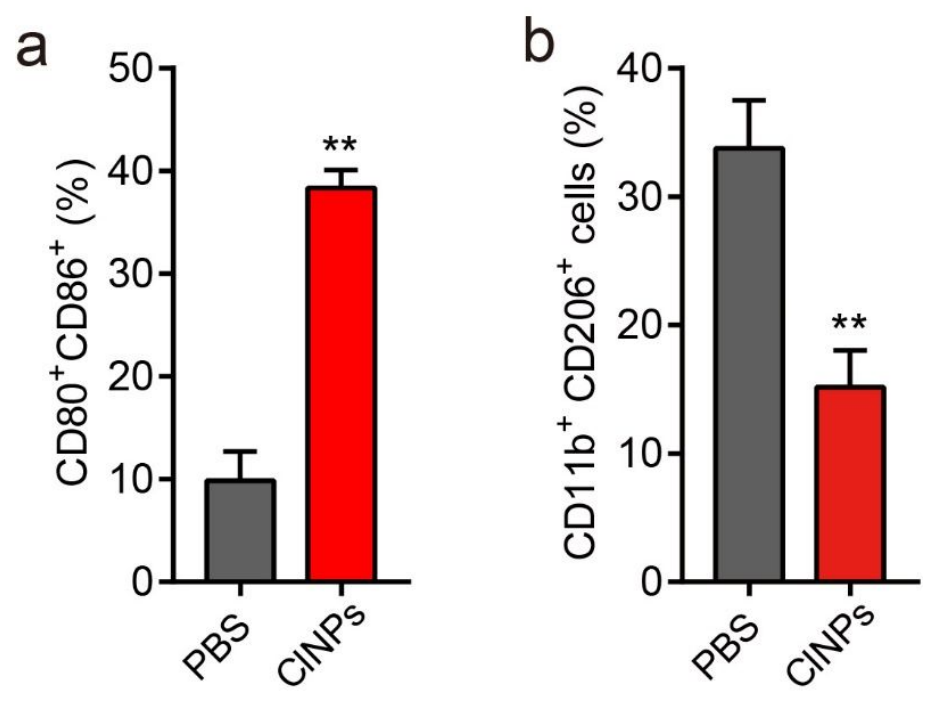

Figure S5. a) Corresponding quantification of $\mathrm{CD} 80^{+} \mathrm{CD} 86^{+}$cells gating on $\mathrm{CD} 11 \mathrm{~b}^{+}$ cells and $\mathrm{CD} 11 \mathrm{~b}^{+} \mathrm{CD} 206^{+}$cells. Statistical significance was calculated using Student's t-test. $* P<0.05, * * P<0.01, * * * P<0.001$ 


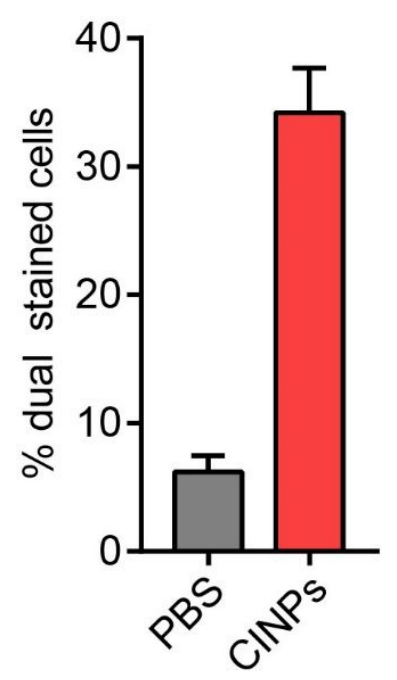

Figure S6. Percentage of phagocytosis were analyzed by measuring the percentage of dual-stained cells. Statistical significance was calculated using Student's t-test. ${ }^{*} P<$ $0.05, * * P<0.01, * * * P<0.001$. 

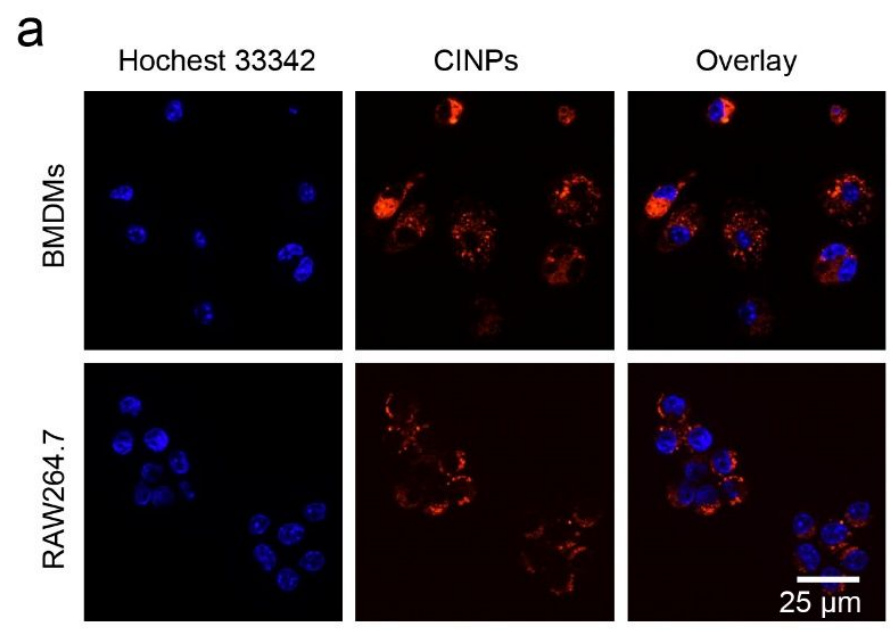

b

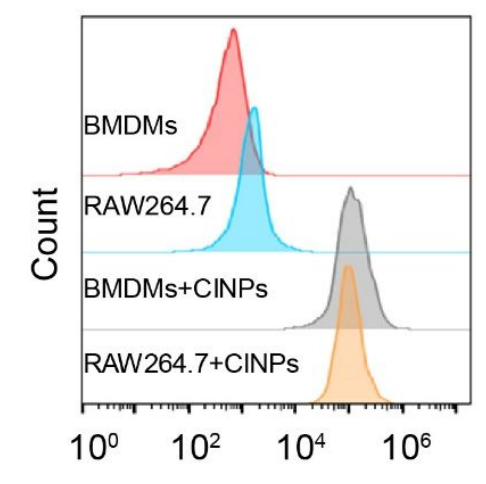

Figure S7. a) Representative confocal images of BMDMs and RAW264.7 cells after treatment with Cy5-labled CINPs, and the nuclei were stained with Hoechst 33342. b) Flow cytometry analysis of intracellular Cy5-labled CINPs after co-culturing for $12 \mathrm{~h}$. 


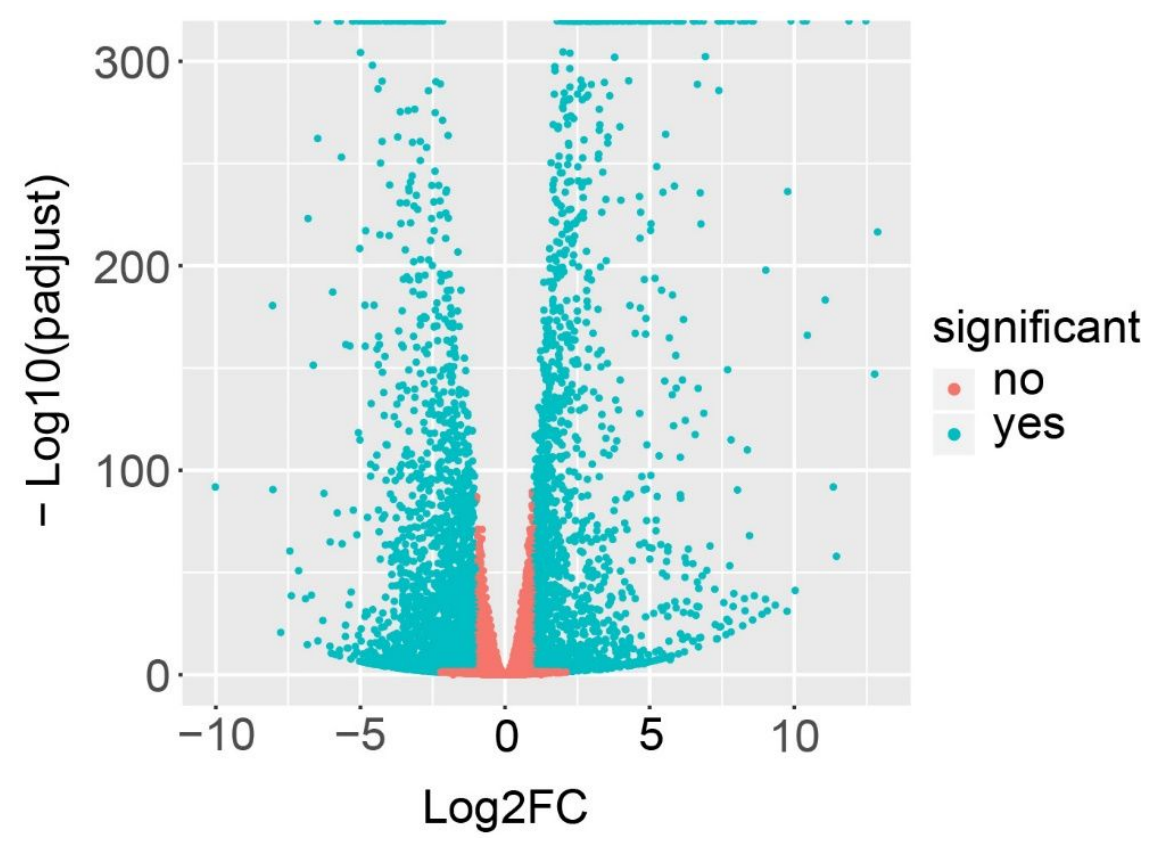

Figure S8. Volcano plots indicating the differentially expressed genes between PBS group and CINPs group in transcriptomics analysis. 


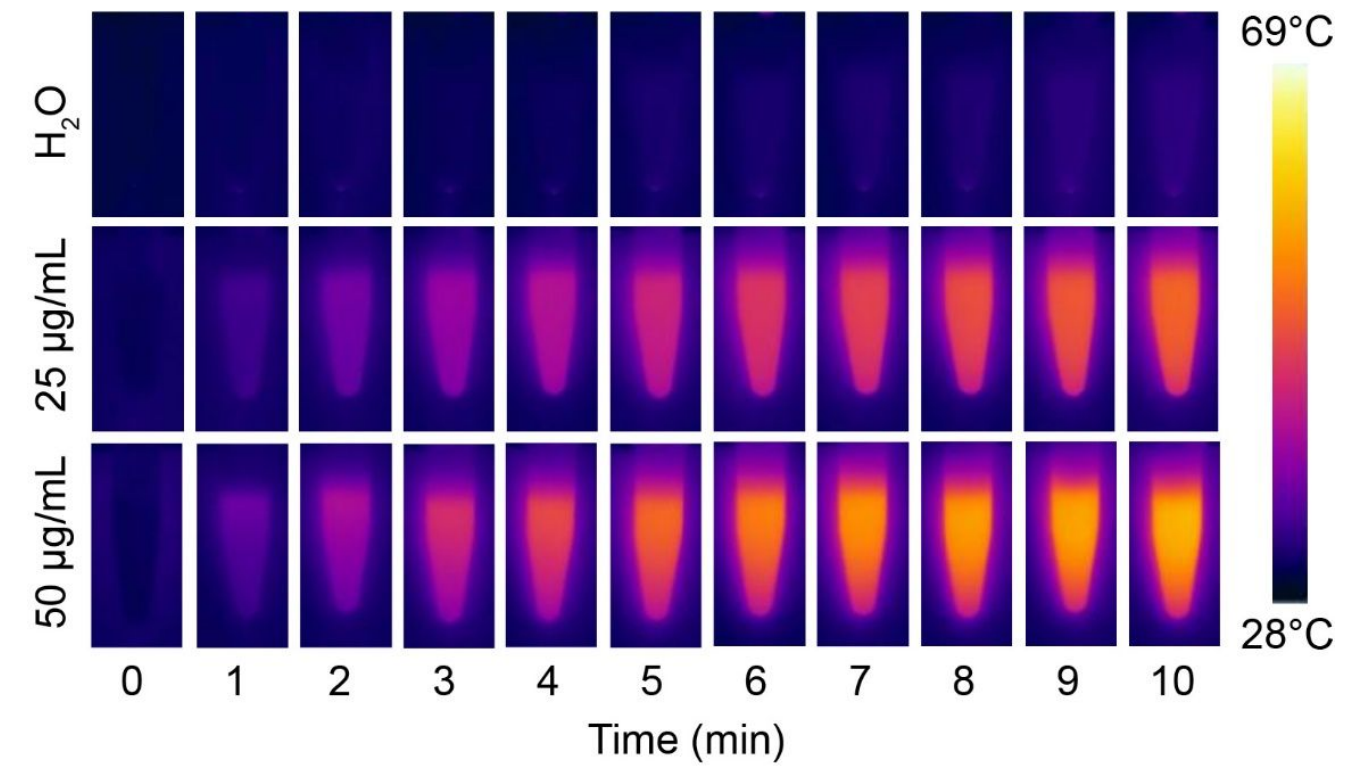

Figure S9. Photothermal images of CINPs with different concentrations. 
Dark-0 $\mu \mathrm{g} / \mathrm{mL}$
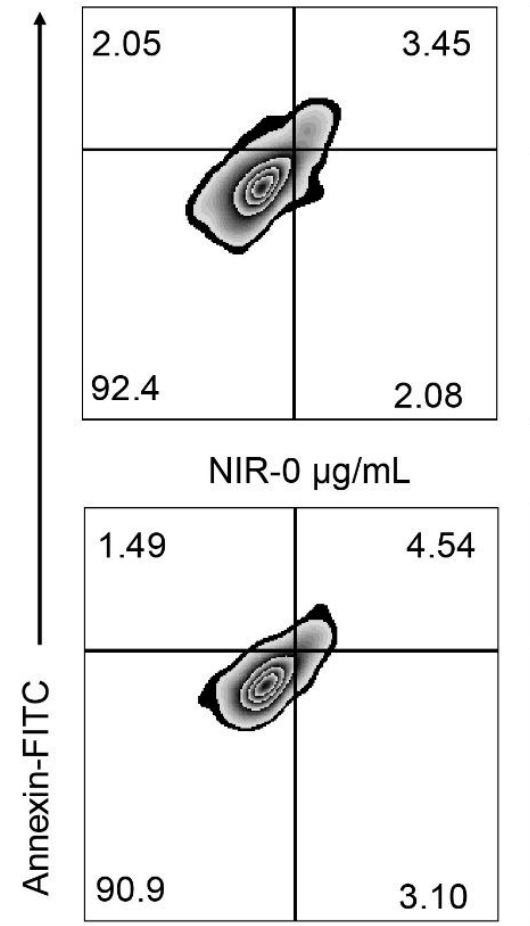

$\mathrm{PI}$
Dark-50 $\mu \mathrm{g} / \mathrm{mL}$
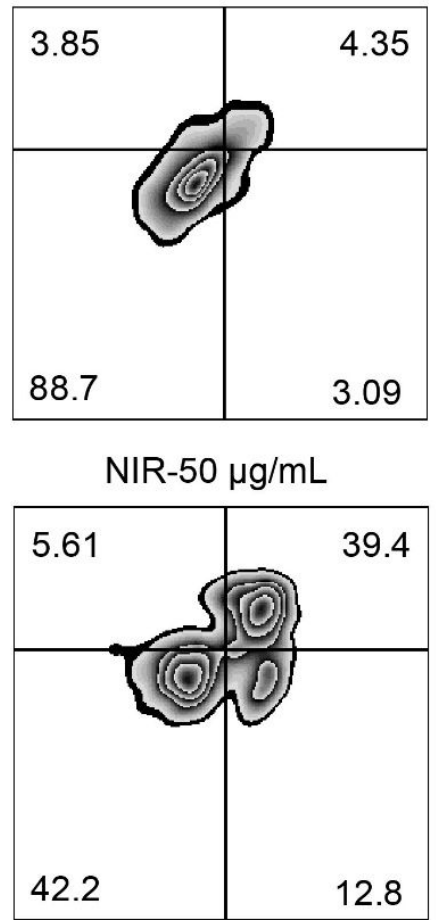

Dark-100 $\mu \mathrm{g} / \mathrm{mL}$

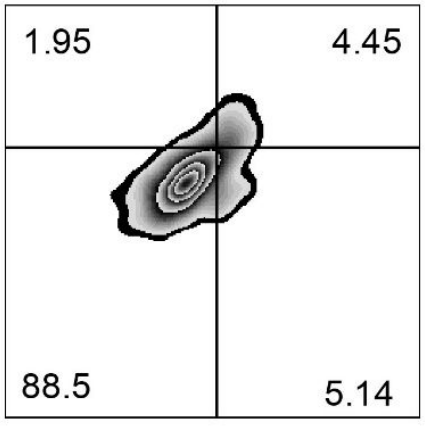

NIR-100 $\mu \mathrm{g} / \mathrm{mL}$

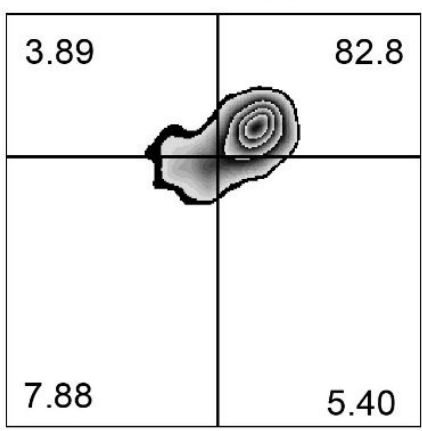

Figure S10. Annexin-FITC/PI labeling flow cytometry analysis of the dark and NIR toxicities under various concentration of CINPs. 
a

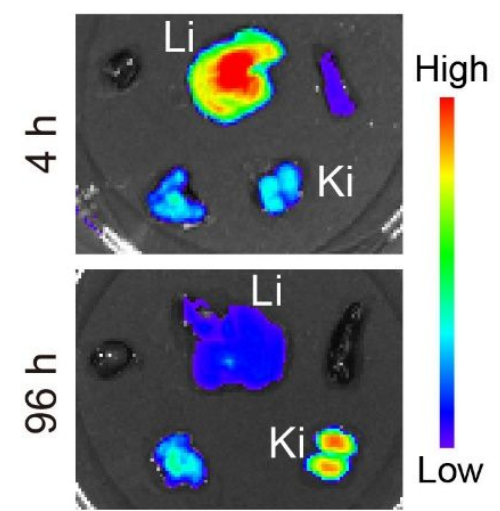

b

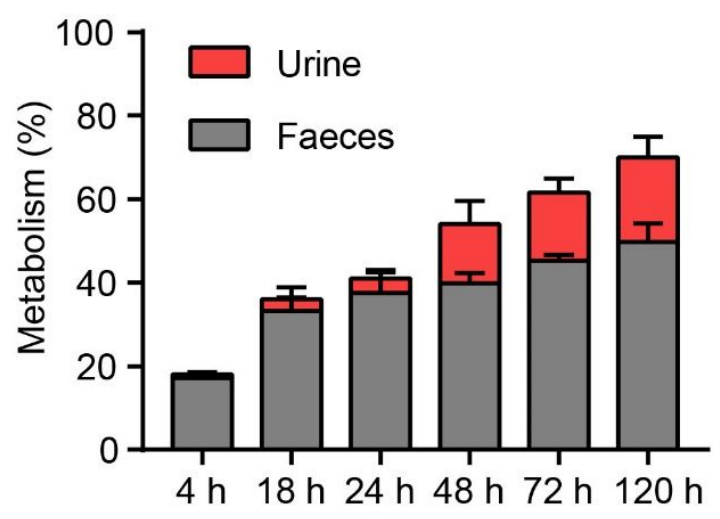

Figure S11. a) The distribution of Cy5-labeled CINPs in different organs. b) Total Cy5labeled CINPs concentration in feces and urine of mice collected after intravenous injection. 


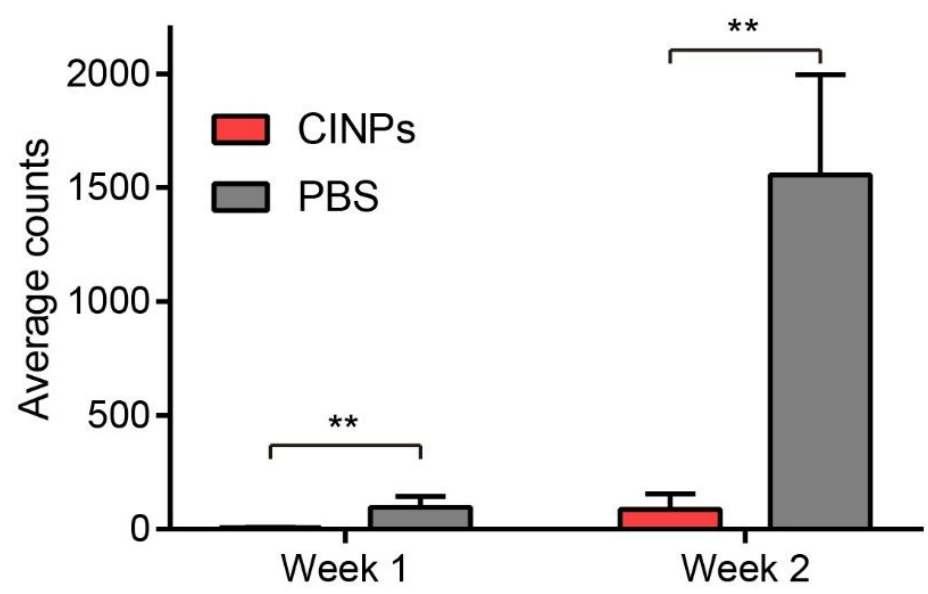

Figure S12. Corresponding quantitative luminescence of PBS-treated and CINPstreated mice in weekly intervals. Statistical significance was calculated using Student's t-test. $* P<0.05, * * P<0.01, * * * P<0.001$. 


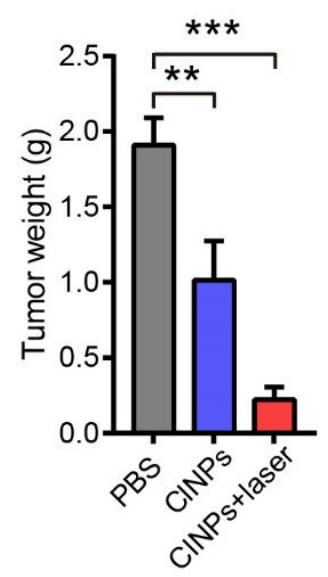

Figure S13. Corresponding average tumor weight obtained on the 16th day after different treatments. Statistical significance was calculated using one-way ANOVA with Tukey post-hoc test. $* P<0.05, * * P<0.01, * * * P<0.001$. 
a
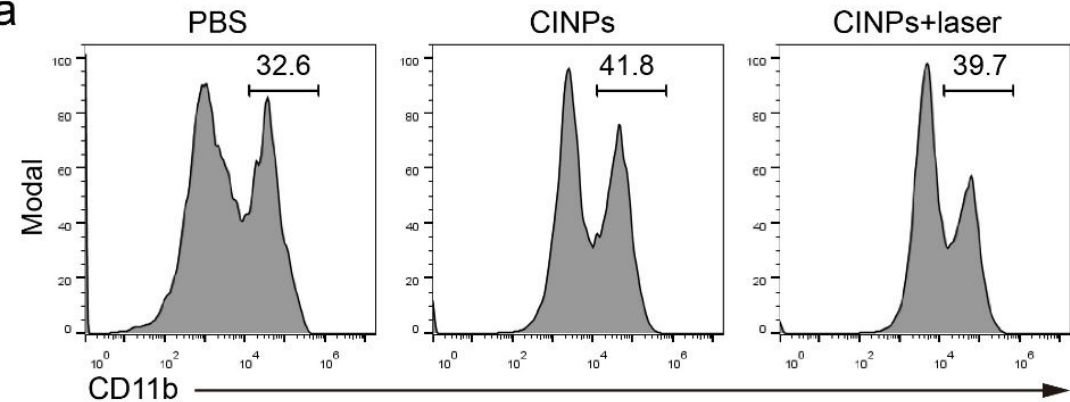

b
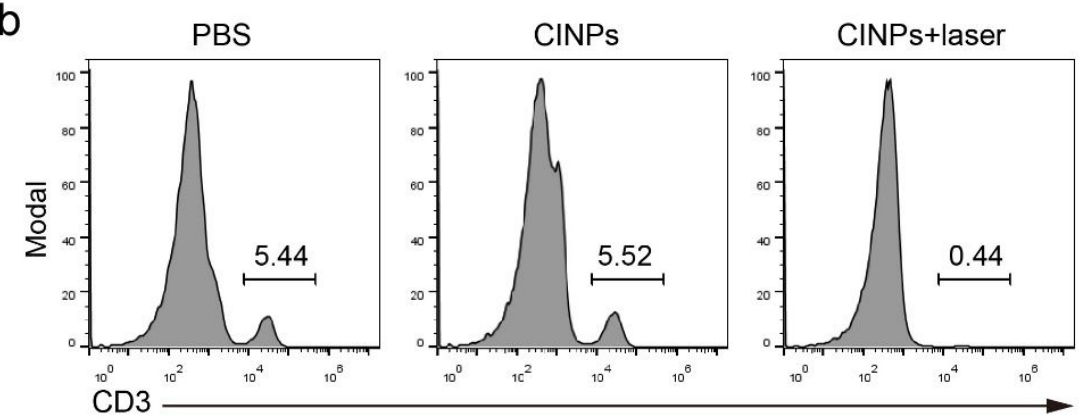
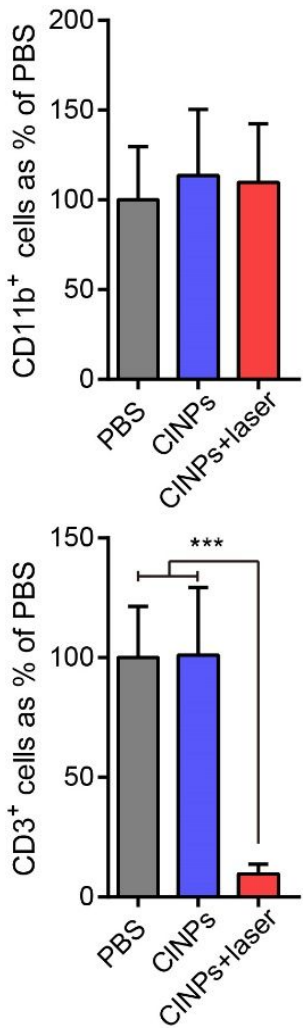

Figure S14. a) Representative flow cytometric analysis images and quantification of $\mathrm{CD} 11 \mathrm{~b}^{+}$cells in primary tumors after different treatments for 3 days. b) Representative flow cytometric analysis images and quantification of $\mathrm{CD}^{+}$cells in primary tumors after different treatments for 3 days. Statistical significance was calculated using oneway ANOVA with Tukey post-hoc test. ${ }^{*} P<0.05,{ }^{*} P<0.01, * * * P<0.001$. 

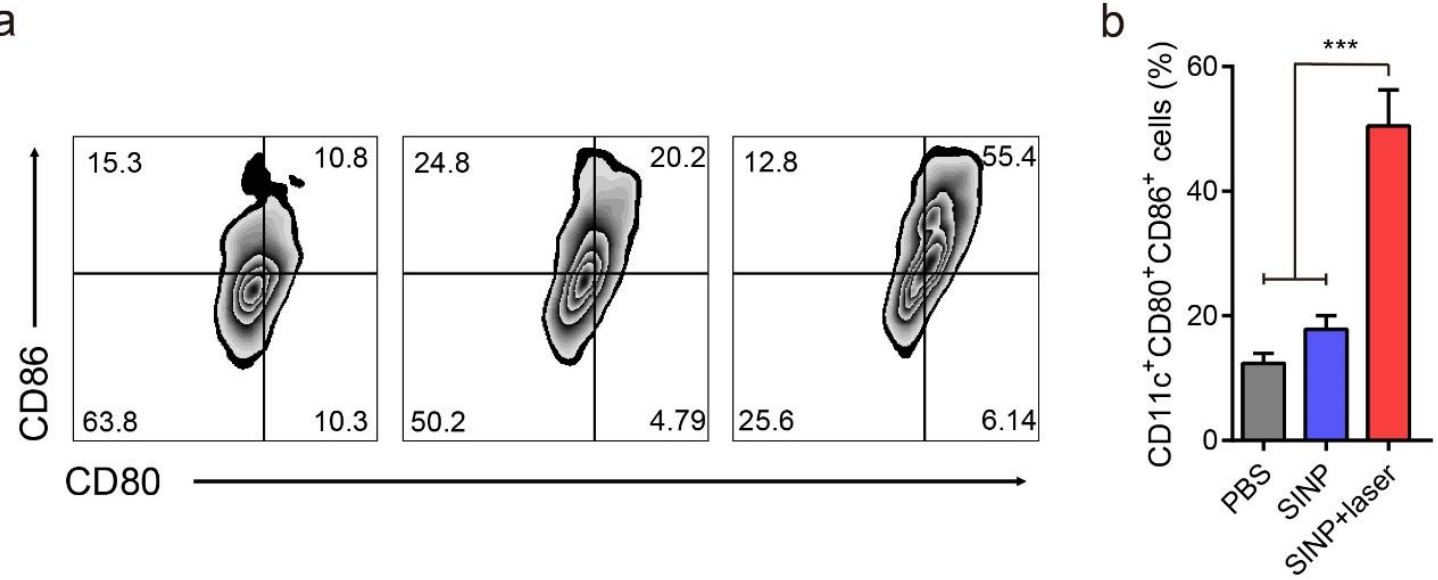

Figure S15. a) Representative flow cytometric analysis images and b) relative quantification show the percentage of $\mathrm{CD} 80^{+} \mathrm{CD} 86^{+}$cells gating on $\mathrm{CD} 11 \mathrm{c}^{+}$cells in primary tumors after different treatments. Statistical significance was calculated using one-way ANOVA with Tukey post-hoc test. ${ }^{*} P<0.05,{ }^{*} P<0.01, * * * P<0.001$. 
a

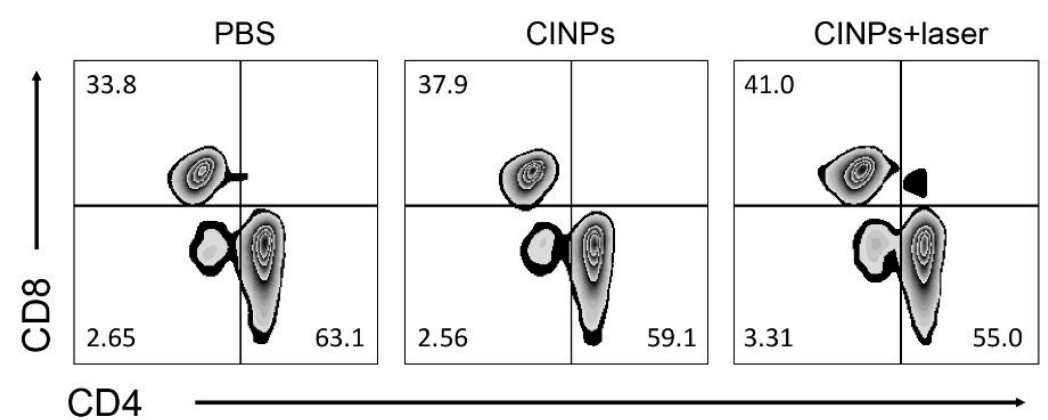

b

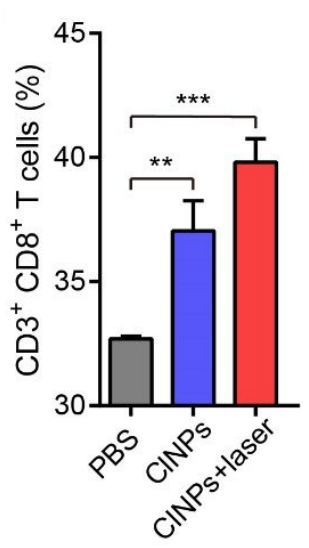

Figure S16. a) Representative flow cytometric analysis images and b) relative quantification show the percentage of $\mathrm{CD}^{+}$and $\mathrm{CD}^{+} \mathrm{T}$ cells gating on $\mathrm{CD} 3^{+}$cells from CT26-bearing mice spleen following different treatments. Statistical significance was calculated using one-way ANOVA with Tukey post-hoc test. $* P<0.05, * * P<$ $0.01, * * * P<0.001$ 

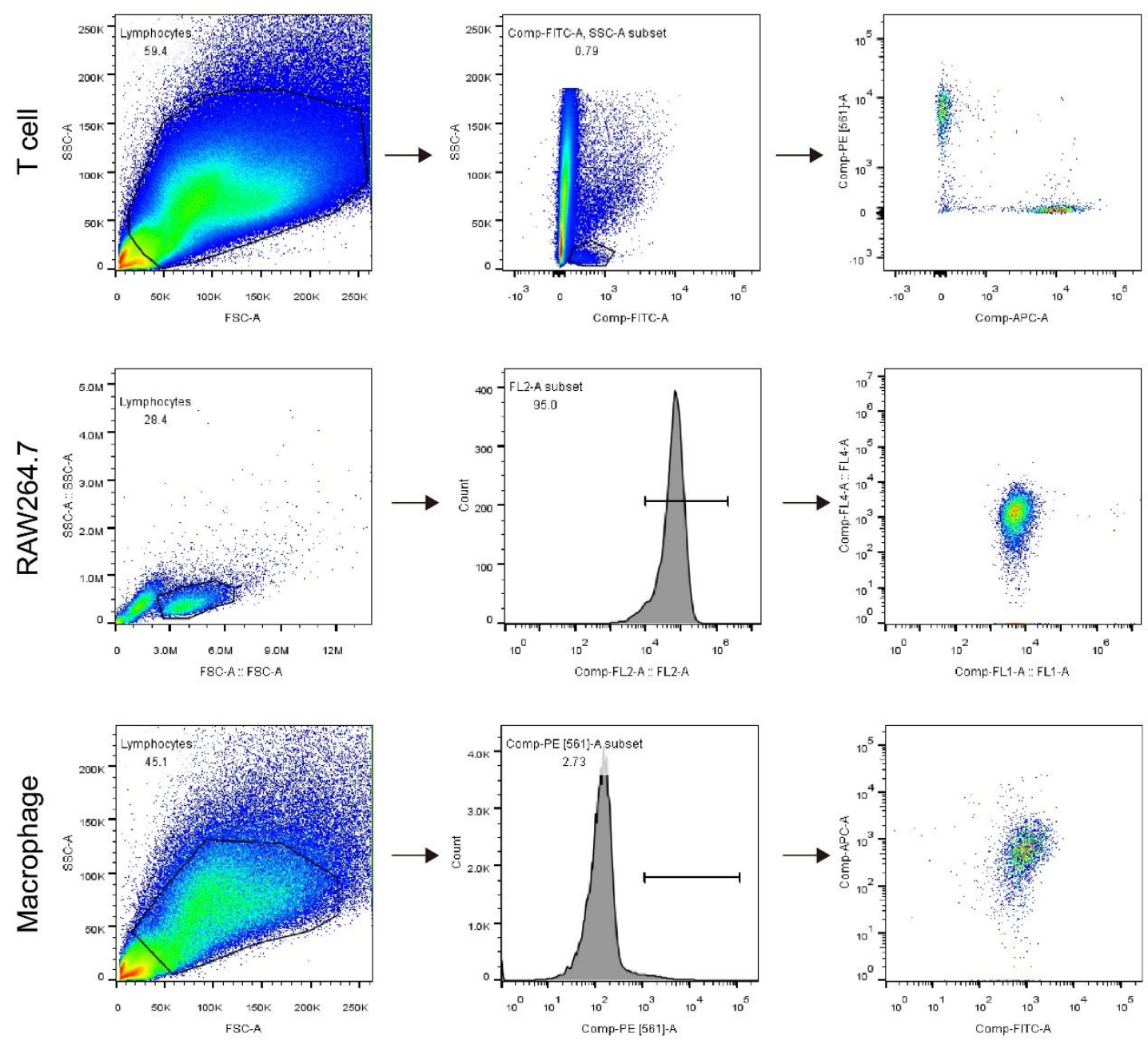

Figure S17. The flow cytometry gating strategy. 
Table S1. Various amino acids content in CINPs

\begin{tabular}{ll}
\hline Amino acid & $\%$ in CINPs \\
\hline Asp & 1.154 \\
Thr & 0.567 \\
Ser & 0.553 \\
Glu & 1.155 \\
Gly & 1.337 \\
Ala & 0.997 \\
Val & 0.507 \\
Met & 0.020 \\
Ile & 0.443 \\
Leu & 0.170 \\
Tyr & 0.668 \\
Phe & 0.186 \\
\hline Pro & 0.303 \\
\hline
\end{tabular}


Table S2. Various monosaccharide in CINPs

\begin{tabular}{ll}
\hline monosaccharide & Content $(\mu \mathrm{g} / \mathrm{g})$ \\
\hline L-arabinose & 2013.860 \\
Xylose & 83.562 \\
L-fucose & 41.753 \\
L-Galactose & 16.137 \\
D-mannose & 10.575 \\
Glucose & 3.521 \\
D-galacturonic acid & 3.288 \\
Glucosamine & 3.466 \\
D-glucuronic acid & 2.534 \\
Galactosamine & 21.589 \\
\hline
\end{tabular}


Table S3. Various metals content in CINPs

\begin{tabular}{ll}
\hline Metal & Content $(\mathrm{ng} / \mathrm{g})$ \\
\hline $\mathrm{Fe}$ & 1965.735 \\
$\mathrm{Cu}$ & 9697.820 \\
$\mathrm{Zn}$ & 8371.327 \\
$\mathrm{Cd}$ & 573.720 \\
$\mathrm{~Pb}$ & 11.606 \\
\hline
\end{tabular}


Table S4. Primer sequences of real-time PCR analysis

\begin{tabular}{lll}
\hline Gene name & Forward primer & Reverse primer \\
\hline CD86 & CTAAGCAAGGTCACCCGAAAC & GGCCACAGTAACTGAAGCTGTAT \\
TNF- $\alpha$ & TCCCCAAAGGGATGAGAAGTT & GAGGAGGTTGACTTTCTCCTGG \\
iNOS & CATTCAGATCCCGAAACGCT & TGTAGGACAATCCACAACTCGC \\
IL-12p40 & GGACATCATCAAACCAGACCC & ACGCACCTTTCTGGTTACACC \\
Arginase-1 & GCATATCTGCCAAAGACATCGT & TCTTCCATCACCTTGCCAATC \\
CD206 & CGTTTCGGTGGACTGTGGA & GTTGTGGGCTCTGGTGGG \\
GAPDH & TGAAGGGTGGAGCCAAAAG & AGTCTTCTGGGTGGCAGTGAT \\
\hline
\end{tabular}

